\title{
Mobility of Spatial Parallel Manipulators
}

\author{
Jing-Shan Zhao Fulei Chu and Zhi-Jing Feng \\ Department of Precision Instruments and Mechanology, \\ Tsinghua University, Beijing 100084, \\ P. R. China
}

\section{Introduction}

This chapter focuses on the mobility analysis of spatial parallel manipulators. It first develops an analytical methodology to investigate the instantaneous degree of freedom (DOF) of the end-effector of a parallel manipulator. And then, the instantaneous controllability of the end-effector is discussed from the viewpoint of the possible actuation schemes which will be especially useful for the designers of the parallel manipulators. Via comparing the differences and essential mobility of a set of underactuated, over actuated and equally actuated manipulators, this chapter demonstrates that the underactuated, over actuated and equally actuated manipulators are all substantially fully actuated mechanisms. This work is significantly important for a designer to contrive his or her manipulators with underactuated or over actuated structures.

Based on the analytical model of the DOF of a spatial parallel manipulator, this chapter develops a general process to synthesize the manipulators with the specified mobility. The outstanding characteristics of the synthesis method are that the whole process is also analytical and each step can be programmed at a computer. Because of the restrictions of the traditional general mobility formulas for spatial mechanisms, a lot of mechanisms having special manoeuvrability might not be synthesized. However, any mechanism can be synthesized with this analytical theory of degrees of freedom for spatial mechanisms.

\section{The valid means to investigate the mobility of a mechanism}

The quick calculation approaches based on the algebra summations of the number of the links, joints and the constraints induced by the joints can not be completely perfected by itself. This is true even the analytical methods are applied in seeking the common constraints (Hunt, 1978)(Waldron, 1966)(Huang, 2006). These problems are becoming more and more obvious with the advent of spatial parallel manipulators. The primary considerations of the designers for the parallel manipulators have been focused on nothing but the mobility of the end-effector and its controllability. Therefore, the concept of general mobility of a mechanism should be divided into two basic concepts-the degree of freedom of the end-effector and the number of actuations needed to control the end-effector. With this regard, this chapter first introduces two primary definitions: 


\section{Definition 1:}

The DOF of an end-effector totally characterizes the motions of the end-effector including the number, type and direction of the independent motions (Zhao et al, 2004a)(Zhao et al, 2006a).

\section{Definition 2:}

The configuration degree of freedom (CDOF) of a mechanism with an end-effector indicates the independent number of actuations required to uniquely control the end-effector under a configuration (Zhao et al, 2004b)(Zhao et al, 2006c).

Obviously, the DOF of an end-effector in number is not larger than 6 but the independent number of actuations required to uniquely control the end-effector might be any nonnegative integer. Bearing the above two definitions in mind, one can fall into two steps to investigate the mobility of a mechanism - the DOF of the end-effector and the CDOF of the mechanism with the prescribed end-effector. The former definition indicates the full instantaneous mobility properties of the end-effector through a mathematics concept of free mobility space while the later one presents the instantaneous controllability of the mechanism system. By definition 1, one can find that the DOF of an end-effector is only subjected to the constraint(s) exerted by the kinematic chain(s) connecting the end-effector with the fixed base or ground. Besides, the degree of freedom of the end-effector, instantaneously associated with the spatial configurations of the kinematic chain(s), should clearly depict the number, the direction and the type of the free motion of the end-effector instantaneously. Therefore, only analytical methods can fulfil such a task.

After obtaining the free motions of the end-effector, an engineering question will naturally arise-how many actuations are needed to control the end-effector? By definition 2, one can find that a checking process is given for verifying the controllability of the mechanism with the specified end-effector. Besides, this process can also allow the different selections of the actuation schemes, which is most adapted to the concept design of a manipulator.

Consequently, the valid means to investigate the mobility of mechanisms can be addressed as: (1) investigate the instantaneous DOF of the prescribed end-effector; and (2) investigate the number of actuations required to uniquely control the end-effector of the mechanism. For the instantaneous characteristics of the mobility of a mechanism, only analytical means is acceptable for such a task. Because of the elegance in depicting the relationship between the motions and the constraints, reciprocal screw theory does be a well selection to accomplish the task. Therefore, the following analytical model for the mobility of a parallel manipulator will be built up by applying the reciprocal screw theory.

According to reciprocal screw theory (Hunt, 1978)(Phillips, 1984)(Phillips, 1990)(Phillips et al, 1964)(Waldron, 1966)(Ball, 1900), a screw $\$$ is defined by a straight line with an associated pitch $h$ and is conveniently denoted by six Plücker homogeneous coordinates:

$$
\$=\left[\begin{array}{c}
\mathrm{s} \\
\mathrm{s}_{0}+h \mathrm{~s}
\end{array}\right]
$$

where $\boldsymbol{s}$ denotes direction ratios pointing along the screw axis, $\boldsymbol{s}_{0}=\boldsymbol{r} \times \boldsymbol{s}$ defines the moment of the screw axis about the origin of the coordinate system, $\boldsymbol{r}$ is the position vector of any point on the screw axis with respect to the coordinate system. Consequently, the screw axis can be denoted by the Plücker homogeneous coordinates $\$_{a x i s}=\left[\begin{array}{c}\mathrm{s} \\ \mathrm{s}_{0}\end{array}\right]$. 
Assume

$$
\left\{\begin{array}{l}
\boldsymbol{s}=\left(\begin{array}{lll}
L & M & N
\end{array}\right)^{T} \\
\boldsymbol{s}_{0}+h \boldsymbol{s}=\left(\begin{array}{lll}
P & Q & R
\end{array}\right)^{T}
\end{array}\right.
$$

Considering $\boldsymbol{s} \cdot\left(\boldsymbol{s}_{0}+h \boldsymbol{s}\right)=\boldsymbol{s} \cdot \boldsymbol{s}_{0}+\|\boldsymbol{s}\|^{2} h=\|\boldsymbol{s}\|^{2} h$ and presuming $\boldsymbol{s} \neq \mathbf{0}$, one obtains the instant pitch of a screw:

$$
h=\frac{\mathbf{s} \cdot\left(\mathbf{s}_{0}+h \mathbf{s}\right)}{\|\mathbf{s}\|^{2}}=\frac{L P+M Q+N R}{L^{2}+M^{2}+N^{2}}
$$

Therefore, the axis of the screw can also be denoted as:

$$
\$_{\text {axis }}=\left(\begin{array}{llllll}
L & M & N & P-L h & Q-M h & R-N h
\end{array}\right)^{T}
$$

Assume that the vector of the projective point of the origin on the screw axis is represented by $\boldsymbol{r}_{O_{P}}$, there will be $\boldsymbol{s} \perp \boldsymbol{r}_{O_{P}}$ and:

$$
\boldsymbol{s} \times\left(\boldsymbol{r}_{O_{P}} \times \boldsymbol{s}\right)=(\boldsymbol{s} \cdot \boldsymbol{s}) \boldsymbol{r}_{O_{P}}-\left(\boldsymbol{s} \cdot \boldsymbol{r}_{O_{P}}\right) \boldsymbol{s}=\|\boldsymbol{s}\|^{2} \boldsymbol{r}_{O_{P}}
$$

According to equations (1) and (2), there are:

$$
\left\{\begin{array}{l}
\boldsymbol{s}=\left(\begin{array}{lll}
L & M & N
\end{array}\right)^{T} \\
\boldsymbol{r}_{O_{P}} \times \boldsymbol{s}=\left(\begin{array}{lll}
P-L h & Q-M h & R-N h
\end{array}\right)^{T}
\end{array}\right.
$$

which yields:

$$
\boldsymbol{r}_{O_{P}}=\frac{\boldsymbol{s} \times\left(\boldsymbol{r}_{O_{P}} \times \boldsymbol{s}\right)}{\|\boldsymbol{s}\|^{2}}=\frac{1}{L^{2}+M^{2}+N^{2}}\left[\begin{array}{c}
M(R-N h)-N(Q-M h) \\
N(P-L h)-L(R-N h) \\
L(Q-M h)-M(P-L h)
\end{array}\right]
$$

Consequently, if the Plücker coordinates of a screw are given, one can easily obtain the unit direction vector, $s$, the pitch, $h$, the screw axis and the vector of the projective point of the origin on the axis, $r_{O_{P}}$, with equations (1) through (7).

If the pitch of a screw equals zero, the screw coordinates reduce to be:

$$
\$=\left[\begin{array}{c}
s \\
s_{0}
\end{array}\right]
$$

which is just the Plücker homogeneous coordinates of the screw axis.

In fact, formula (8) uniquely defines a line in a three-dimensional space. Assume that point $O_{P}$ is the projective point of the origin on a line $l$ and point $A$ is any other point on the line. Then, 


$$
\boldsymbol{r}_{A}=\boldsymbol{r}_{O_{P}}+\boldsymbol{r}_{O_{P} A}=\boldsymbol{r}_{O_{P}}+\frac{a s}{\|s\|}
$$

where $\boldsymbol{s}$ is a direction vector of line $l, a$ is the length of line segment $O_{P} A$.

The moment of line $l$ about the origin at point $A$ will be:

$$
\boldsymbol{s}_{0}=\boldsymbol{r}_{A} \times \boldsymbol{s}=\left(\boldsymbol{r}_{O_{P}}+\frac{a \boldsymbol{s}}{\|\boldsymbol{s}\|}\right) \times \boldsymbol{s}=\boldsymbol{r}_{O_{P}} \times \boldsymbol{s}
$$

From equations (9) and (10), one obtains that the moment of a line about the origin is irrelevant to the point's selection on the line.

If a screw passes through the origin of the coordinate system, the screw coordinates can be denoted as:

$$
\boldsymbol{\$}=\left[\begin{array}{c}
\boldsymbol{s} \\
h \boldsymbol{s}
\end{array}\right]
$$

On the other hand, if the pitch of a screw is infinite, the screw is defined as:

$$
\boldsymbol{S}=\left[\begin{array}{l}
\mathbf{0} \\
\boldsymbol{s}
\end{array}\right]
$$

where $\mathbf{0}=\left(\begin{array}{lll}0 & 0 & 0\end{array}\right)^{T}$ is a three dimensional vector.

According to the above definitions, a screw associated with a revolute pair is a twist of zero pitch pointing along the pair axis while a screw associated with a prismatic pair is a twist of infinite pitch pointing in the direction of the translational guide line of the pair.

From equation (11), one has known that the kinematic screw is often denoted in the form of Plücker homogeneous coordinates:

$$
\$=\left(\begin{array}{llllll}
L & M & N & P & Q & R
\end{array}\right)^{T}
$$

where the first three components denote the angular velocity, the last three components denote the linear velocity of a point in the rigid body that is instantaneously coincident with the origin of the coordinate system.

Similarly, $\boldsymbol{s}^{r}$ is defined as:

$$
\$^{r}=\left(\begin{array}{llllll}
L^{r} & M^{r} & N^{r} & P^{r} & Q^{r} & R^{r}
\end{array}\right)^{T}
$$

where the first three components denote the resultant force and the last three components denote the resultant moment about the origin of the coordinate system.

Two screws, $\$$ and $\$^{r}$, are called to be reciprocal if they satisfy the equation:

$$
L P^{r}+M Q^{r}+N R^{r}+P L^{r}+Q M^{r}+R N^{r}=0
$$


Obviously, the free motions (general twists) $\$$ and the prescribed constraints (general wrenches) $\$^{r}$ of an equilibrium rigid body should satisfy equation (15). Equation (15) is often written for short (Kumar, 1992):

$$
(\$)^{T} E \$^{r}=0
$$

where $\$$ and $\$^{r}$ are column vectors, $E=\left[\begin{array}{ll}\mathbf{0}_{3} & \mathbf{I}_{3} \\ \mathbf{I}_{3} & \mathbf{0}_{3}\end{array}\right]$, and $\mathbf{I}_{3}$ and $\mathbf{0}_{3}$ are $3 \times 3$ identity and zero matrices, respectively.

Similarly, if one gets a set of terminal constraints exerted to a rigid body, its free motion(s) can also be solved through equation (16). Next, one can investigate the instantaneous mobility of the end-effector of a parallel manipulator with equation (16).

\subsection{The degree of freedom of the end-effector of a parallel manipulator}

The free motions of the end-effector can be instantaneously expressed in a set of Plücker homogeneous coordinates in one Cartesian coordinate system. The main steps are:

\section{Investigate the Terminal Constraints of the Kinematic Chains}

In general, any parallel manipulator can be decomposed into $n(n \geq 1)$ kinematic chains connecting the end effector with the base. In order to instantaneously analyze the mobility properties of the end-effector, this section only establishes one absolute coordinate system. After establishing the coordinate system, the Plücker homogeneous coordinates of all kinematic pairs in a chain can be obtained. Group all of the kinematic screws of the same chain to be $\$_{i}(i=1,2, \cdots, n)$ and solve the terminal constraint(s) $\$_{i}^{r}$ with equation (16).

In fact, if all of the terminal constraints of the kinematic chains are gained, the constraints exerted to the end-effector, denoted by $\boldsymbol{\$}_{E}^{\mathrm{r}}$, should also be obtained. The dimension of constraint spaces spanned by the terminal constraints of kinematic chains can be simplified as $d=\operatorname{Rank}\left(\boldsymbol{\$}_{E}^{\mathrm{r}}\right)$.

\section{Solve the Free Motion(s), $\$_{E}^{F}$, of the End-Effector with Equation (16)}

Naturally, the mobility properties of the end-effector is fully expressed by $\$_{E}^{\mathrm{F}}$. Its number of DOF can be expressed as:

$$
M=\operatorname{Rank}\left(\$_{E}^{\mathrm{F}}\right)=6-d
$$

Now, the DOF of the end-effector of the parallel manipulator shown in Fig. 1 can be instantaneously investigated with the above two steps. In this manipulator, the end-effector $C_{1} C_{2} C_{3}$ has three identical PPRR kinematic chains connected with the fixed base. For the sake of modelling, one can establish any Cartesian coordinate system for the manipulator. Assume that the direction vector of the prismatic joint $A_{i}(i=1,2,3)$ is denoted by $e_{A_{i}}=\left(\begin{array}{lll}a_{i 1} & b_{i 1} & c_{i 1}\end{array}\right)^{T}$, the direction vector of the prismatic joint $B_{i}(i=1,2,3)$ is denoted by 
$e_{B_{i}}=\left(\begin{array}{lll}a_{i 2} & b_{i 2} & c_{i 2}\end{array}\right)^{T}$, the rotational vector of the revolute joint $B_{i}$ is denoted by $e_{B_{i}^{r}}=e_{A_{i}} \times e_{B_{i}}=\left(\begin{array}{lll}b_{i 1} c_{i 2}-b_{i 2} c_{i 1} & a_{i 2} c_{i 1}-a_{i 1} c_{i 2} & a_{i 1} b_{i 2}-a_{i 2} b_{i 1}\end{array}\right)^{T}$, the rotational vector of the revolute joint $C_{i}(i=1,2,3)$ is denoted by $e_{C_{i}^{r}}=e_{B_{i}^{r}}$. Also suppose that $e_{A_{1}} \neq e_{A_{2}} \neq e_{A_{3}}$ and $e_{B_{i}} \cdot e_{A_{i}}=0$.

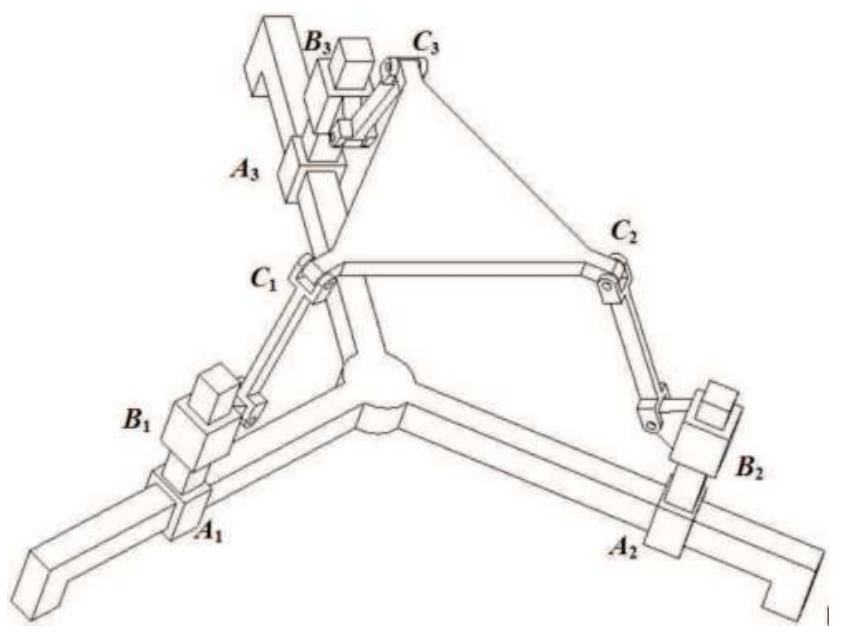

Fig. 1 a 3-PPRR Spatial Mechanism

So, the kinematic screws for each kinematic chain can be expressed as:

$$
\$_{A_{i} B_{i} C_{i}}=\left[\begin{array}{llll}
\$_{A_{i}} & \$_{B_{i}} & \$_{B_{i}^{r}} & \$_{C_{i}^{r}}
\end{array}\right]
$$

where $\$_{A_{i}}=\left(\begin{array}{llllll}0 & 0 & 0 & a_{i 1} & b_{i 1} & c_{i 1}\end{array}\right)^{T}, \$_{B_{i}}=\left(\begin{array}{llllll}0 & 0 & 0 & a_{i 2} & b_{i 2} & c_{i 2}\end{array}\right)^{T}$,

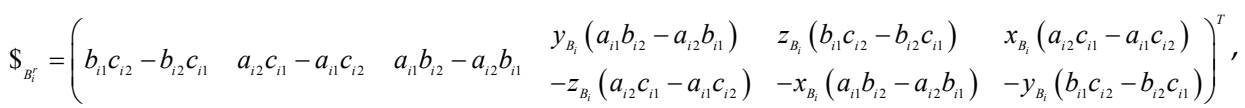

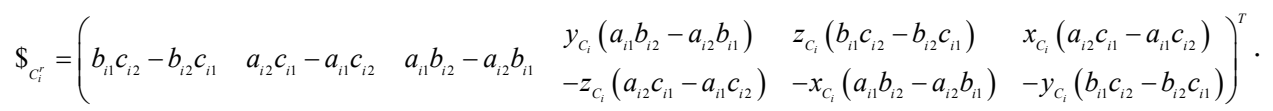

The terminal constraints of the kinematic chain can be solved with (16):

$$
\boldsymbol{\$}_{A_{i} B_{i} C_{i}}^{r}=\left[\begin{array}{lll}
\boldsymbol{\$}_{i}^{r_{1}} & \boldsymbol{\$}_{i}^{r_{2}} & \boldsymbol{\$}_{i}^{r_{3}}
\end{array}\right]
$$

where $\$_{i}^{r_{1}}=\left(\begin{array}{llllll}0 & 0 & 0 & a_{i 1} & b_{i 1} & c_{i 1}\end{array}\right)^{T}, \$_{i}^{r_{2}}=\left(\begin{array}{llllll}0 & 0 & 0 & a_{i 2} & b_{i 2} & c_{i 2}\end{array}\right)^{T}$,

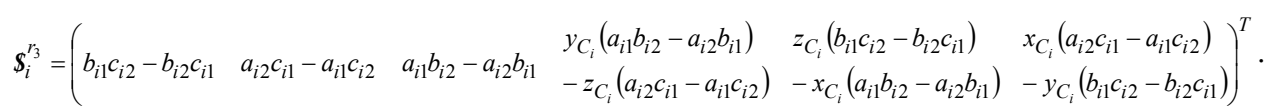


According to the mechanism shown in Fig. 1, $e_{B_{1}}=e_{B_{2}}=e_{B_{3}}$. Therefore, the terminal constraints exerted to the end-effector by these three kinematic chains are:

$$
\boldsymbol{\$}_{C_{1} C_{2} C_{3}}^{r}=\left[\begin{array}{lllllll}
\boldsymbol{\$}_{1}^{r_{1}} & \boldsymbol{\$}_{2}^{r_{1}} & \boldsymbol{\$}_{3}^{r_{1}} & \boldsymbol{\$}_{1}^{r_{2}} & \boldsymbol{\$}_{1}^{r_{3}} & \boldsymbol{\$}_{2}^{r_{3}} & \boldsymbol{\$}_{3}^{r_{3}}
\end{array}\right]
$$

It is not difficult to find that the rank of $\$_{C_{1} C_{2} C_{3}}^{r}$ expressed by equation (20) is 5 , and the free motions of the end-effector $C_{1} C_{2} C_{3}$ can be again solved with equation (16):

$$
\$_{C_{1} C_{2} C_{3}}^{F}=\left(\begin{array}{llllll}
0 & 0 & 0 & a_{12} & b_{22} & c_{32}
\end{array}\right)^{T}
$$

Equation (21) indicates that the end-effector has one translational DOF along the direction vector $e=\left(\begin{array}{lll}a_{12} & b_{22} & c_{32}\end{array}\right)^{T}$. Of course, the number of the DOF of the end-effector is $\operatorname{Rank}\left(\$_{C_{1} C_{2} C_{3}}^{F}\right)=1$, the direction is $e=\left(\begin{array}{lll}a_{12} & b_{22} & c_{32}\end{array}\right)^{T}$ and the type is translation, which is fully represented by the screw expression (21).

\subsection{The number of actuations required to control the end-effector of a spatial parallel manipulator}

After obtaining the instantaneous mobility of the end-effector, one can directly exert $M$ actuations to the manipulator, and then investigate the CDOF of the end-effector by solving the free motion(s) of the end-effector within its workspace. If the newly solved motion(s), denoted by $\$_{E}^{\mathrm{F}_{\mathrm{ni}}}, i=1,2, \cdots$, satisfy that $\boldsymbol{\$}_{E}^{\mathrm{F}_{\mathrm{ni}}} \neq\left(\begin{array}{llllll}0 & 0 & 0 & 0 & 0 & 0\end{array}\right)^{T}$, then, additional actuations are needed under this configuration and the actuation scheme. Of course, we can either reselect the actuation scheme or add $\operatorname{Rank}\left(\boldsymbol{\$}_{E}^{\mathrm{F}_{\mathrm{ni}}}\right)$ more actuation(s) under this configuration until $\boldsymbol{\$}_{E}^{\mathrm{F}_{\mathrm{ni}}}=\left(\begin{array}{llllll}0 & 0 & 0 & 0 & 0 & 0\end{array}\right)^{T}$. The total number of actuations under the configuration with this actuation scheme is the CDOF. However, what must be pointed out is that the actuation(s) should not be exerted to the joint when the newly increased terminal constraint can be transformed by the other actuation(s). Otherwise, the over constraint case will occur. When there are a lot of possible actuation schemes any one of which can be selected to set the actuators, the controllability of the manipulator is also affected by the actuation scheme's selection. For instance, one can analyze the number of actuation(s) required to control the end-effector of the parallel manipulator shown in Fig. 1. Because the number of DOF of the end-effector is 1, it is reasonable for us to expect that the end-effector can be fully controlled only with one actuation. If one actuation is exerted to any joint of the mechanism, $A_{1}$ for an example, it is not difficult to find that the end-effector still remains one translational DOF in the direction $e=\left(\begin{array}{lll}a_{11} & b_{11} & c_{11}\end{array}\right)^{T}$ when one repeats the above two steps in section 2.1. Therefore, one has to add another actuation to the mechanism. Of course, he can add the second actuation to any one of the rest joints. However, it is not difficult to prove that the 
end-effector will not be controlled unless the second actuation is exerted to the prismatic joint $B_{i}(i=1,2,3)$ under the condition that the first actuation is exerted to $A_{i}(i=1,2,3)$.

However, just as mentioned above, the new-added actuation should not be accepted if the newly-increased terminal constraint can be obtained by translating the former actuation(s). For an example, if the second actuation is assigned to the revolute joint $B_{1}$, the newlyincreased terminal constraints of the kinematic chain $A_{1} B_{1} C_{1}$ will be:

$$
\$_{1}^{r_{n}}=\left(\begin{array}{llllll}
a_{11} & b_{11} & c_{11} & y_{C_{1}} c_{11}-z_{C_{1}} b_{11} & z_{C_{1}} a_{11}-x_{C_{1}} c_{11} & x_{C_{1}} b_{11}-y_{C_{1}} a_{11}
\end{array}\right)^{T}
$$

Equation (22) is the transformation of the actuation exerted to the prismatic joint $A_{1}$. So, the newly-added actuation is an over actuation for the actuation scheme whose first actuation is assigned to $A_{1}$.

Of course, one can also exert the second actuation to the prismatic joint $A_{2}$ after assigning the first actuation to the prismatic joint $A_{1}$. Again, one can find that the end-effector still has

the free translation in the direction $e=\left(\begin{array}{lll}a_{12} & b_{22} & c_{32}\end{array}\right)^{T}$ when one repeats the above two steps in section 2.1. So, one can continue to add the third actuation to the prismatic joint $A_{3}$. However, the end-effector will not be controlled until a fourth actuation is applied to one of the prismatic joints, $B_{1}, B_{2}$ and $B_{3}$. This forms a second actuation scheme. So, under this actuation scheme, the number of actuations needed to control the end-effector shown in Fig. 1 is 4 .

The differences between the second actuation scheme and the first one are that the second one not only completely control the end-effector but also completely control every link in the manipulator. The selections of different actuation schemes can be well accomplished by a computer especially when the possible selections are numerous such as the one shown in Fig. 1. Unfortunately, this properties of a mechanism is ignored by the general mobility formulas.

\section{The substantial mobility of underactuated, over actuated and equally actuated manipulators}

A manipulator is said to be underactuated when the number of actuators in the manipulator is smaller than the number of degrees of freedom of the mechanism (Laliberte \& Gosselin, 1998). When applied to mechanical fingers, the concept of underactuation leads to shape adaptation, i.e. underactuated fingers will envelope the objects to be grasped and adapt to their shape although each of the fingers is controlled by a reduced number of actuators (Laliberté \& Gosselin, 1998). The concept of underactuation in robotic fingers - with fewer actuators than the degrees of freedom-allows the hand to adjust itself to an irregularly shaped object without complex control strategy and sensors (Birglen \& Gosselin, 2006a). These underactuated manipulators arise in a number of important applications such as space robots, hyper redundant manipulators, manipulators with structural flexibility, etc (Jain \& Rodriguez, 1993). The fact that the underactuated robotic fingers allow the hand to adjust itself to an irregularly shaped object makes it possible that no complex control strategy or numerous sensors are necessary in these manipulators (Birglen \& Gosselin, 
2006b). However, the over actuated mechanical systems often occur in biomechanical systems during the contact with ground and is recently introduced in redundantly actuated parallel robots. Yi and Kim (Yi \& Kim, 2002) designed a singularity free load-distribution scheme for a redundantly actuated three-wheeled Omnidirectional mobile robot. The most outstanding advantage of the redundantly actuated mobile robot is that the singularities of the mechanism can be well avoided. Yiu and Li (Yiu \& Li, 2003) investigated the trajectory generation for an over actuated parallel manipulator, in which there is one redundant actuator. Of course, the redundant actuator(s) and the required actuator(s) must obey a certain relationship determined by the mechanism, which will be discussed in section 3.2.

This section aims at clarifying the substantial relationships between the underactuated, over actuated and the equally actuated manipulators. The underactuated manipulator, which is also called under-determinate input system, means that the number of actuations provided is less than that is necessary; while the over actuated manipulator, which is also called redundant actuation or redundant input system, means that the number of actuations provided is larger than that is necessary. Equally actuated manipulator, which is also called fully actuated or determinate system, means that the actuations provided is equal to that is needed.

From the viewpoint of mechanisms, this classification of manipulators seems to be reasonable and has been widely used in engineering. However, it is not a properly scientific categorization for mechanisms. Therefore, this section will briefly study the substantial relationships between the underactuated, over actuated and equally actuated manipulators that are easily misunderstood in engineering applications.

\subsection{The essence of the underactuated manipulator}

To begin with this section, one might first investigate a famous inverted pendulum system shown in Fig. 2, which is also a representative, underactuated mechanical system. This inverted pendulum system is a planar two degrees of freedom catenation mechanical system. The vehicle can only make reciprocal translation along the $x$-axis and the pendulum can only rotate about the pivot attached to the moving vehicle.

In applications, only one actuation is provided to control the system, which seems to conflict with the definition of a fully actuated mechanism. In order to reveal the essence of this puzzling phenomenon, one might first turn to analyze the dynamics of this two-degree-offreedom system.

Suppose the mass of the vehicle is denoted by $M$, the mass of the pendulum is $m$ and the distance from the pivot attached to the vehicle to the mass center of the pendulum is $l$ and the moment of inertia of the pendulum is denoted by $J$. The dynamics of the system can be immediately established via Lagrange method. The kinetic energy of the vehicle is:

$$
T_{v}=\frac{1}{2} M \dot{x}^{2}
$$

where $T_{v}$ represents the kinetic energy of the vehicle.

The kinetic energy of the pendulum is:

$$
T_{p}=\frac{1}{2} m\left[\frac{d}{d t}(x+l \sin \theta)\right]^{2}+\frac{1}{2} m\left[\frac{d}{d t}(l \cos \theta)\right]^{2}+\frac{1}{2} J \dot{\theta}^{2}
$$

where $T_{p}$ represents the kinetic energy of the pendulum. 


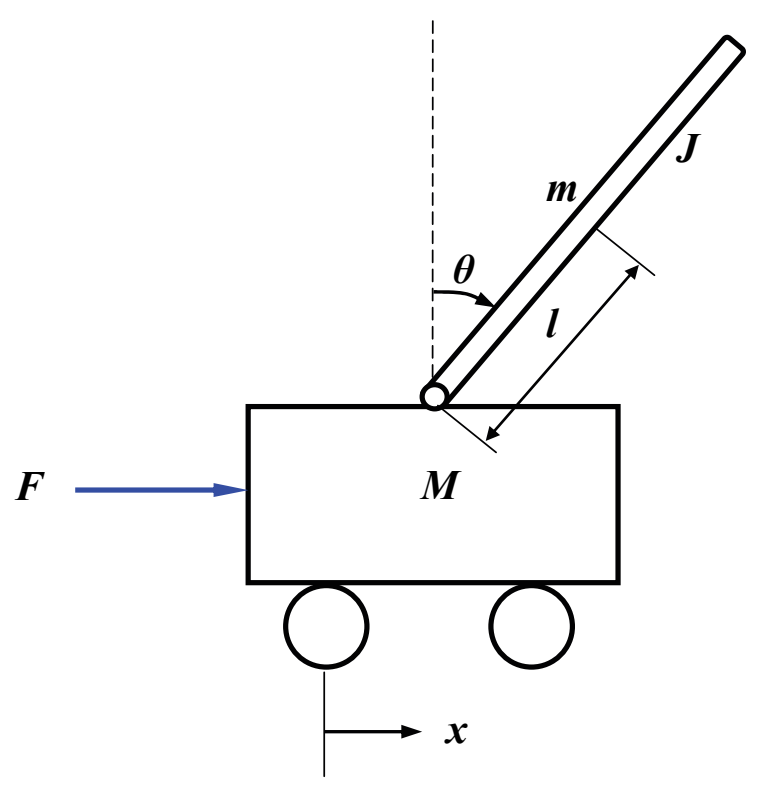

Fig. 2 a single inverted pendulum system

The total kinetic energy of the system is:

$$
T=T_{v}+T_{p}=\frac{1}{2}(M+m) \dot{\bullet}^{2}+m l \dot{x} \dot{\theta} \cos \theta+\frac{1}{2}\left(J+m l^{2}\right) \dot{\theta}^{2}
$$

The potential energy of the system is:

$$
V=m g l \cos \theta
$$

Therefore, the Lagrange function of the system is:

$$
L=\frac{1}{2}(M+m) \dot{\bullet}^{2}+m l \dot{x} \dot{\theta} \cos \theta+\frac{1}{2}\left(J+m l^{2}\right) \dot{\theta}^{2}-m g l \cos \theta
$$

where $L$ indicates the Lagrange function.

The dynamics equations for the two-degree-of-freedom system shown in Fig. 2 can be expressed as:

$$
\left\{\begin{array}{l}
\frac{d}{d t}\left(\frac{\partial L}{\partial \dot{x}}\right)-\frac{\partial L}{\partial x}=F \\
\frac{d}{d t}\left(\frac{\partial L}{\partial \dot{\theta}}\right)-\frac{\partial L}{\partial \theta}=\tau
\end{array}\right.
$$

where $\tau$ represents the torque exerted to the revolute joint that connect the inverse pendulum and the vehicle. 
From the Lagrange function, one immediately obtains:

$$
\begin{gathered}
\frac{\partial L}{\partial \dot{x}}=(M+m) \dot{x}+m l \dot{\theta} \cos \theta, \frac{\partial L}{\partial x}=0 \\
\frac{\partial L}{\partial \dot{\theta}}=m l \dot{x} \cos \theta+\left(J+m l^{2}\right) \dot{\theta}, \frac{\partial L}{\partial \theta}=m l(g-\dot{x} \dot{\theta}) \sin \theta
\end{gathered}
$$

Substituting the above equations into equation (24), one has:

$$
\left\{\begin{array}{l}
(M+m) \ddot{x}-m l \dot{\theta}^{2} \sin \theta+m l \ddot{\theta} \cos \theta=F \\
\ddot{m} \dot{x} \cos \theta+\left(J+m l^{2}\right) \ddot{\theta}-m g l \sin \theta=\tau
\end{array}\right.
$$

Of course, in the underactuated condition, there is $\tau=0$. The first formula in equation set (25) is the apparent actuation formula while the second one in equation set (25) is a hidden relationship of the mechanical system, in which the gravity, the inertia force and moment of the pendulum are associated precisely. As a matter of fact, therefore, this relationship depicted by the second formula in equation set (25) provided another actuation constraint for the two-degrees-of-freedom mechanical system in dynamics but not in statics. Therefore, the mechanical system shown in Fig. 2 is fully actuated in dynamics but not in statics. When

$\ddot{x}=0$ and $\tau=0$, equation set (25) can be simplified as:

$$
\dot{\theta}= \pm \sqrt{\frac{m g l}{J+m l^{2}} \cos \theta-\frac{F}{m l \sin \theta}}
$$

where " \pm " is determined by the initial condition of the system and the sign should be " + " in the case shown in Fig. 2.

From equations (25) and (26), it is not difficulty to find that the inverted pendulum system shown in Fig. 2 can only keep a dynamic equilibrium but not a static equilibrium which is a primary requirement for a mechanism.

A much more familiar example is the differential gear train mechanism used in the driving axle of all kinds of automobiles. The basic mechanism structure is shown in Fig. 3. The pinion gear transforms the torque from the engine to the driving axle shafts by a differential gear train mechanism, in which the ring gear shown in Fig. 3 acts as an actuator and the right and the left shafts act as executors.

Obviously, this mechanism also has two degrees of freedom. However, the actuation is just one rotational input from the pinion gear. One might draw a conclusion in haste that this mechanism should be an outstanding representative example for the applications of underactuated mechanical systems because it is so widely used in the modern vehicles. This mechanical system is really quite different from the inverted pendulum system shown in Fig. 2 in that the hidden mechanical constraint or "actuation" is more easily ignored. The reaction difference between the right and left wheels from the road surface provides such an 
"actuation", which is apparent when the reactions to the right and left wheels from the road surface are different, and which often occurs when the vehicle makes a right or left turn.

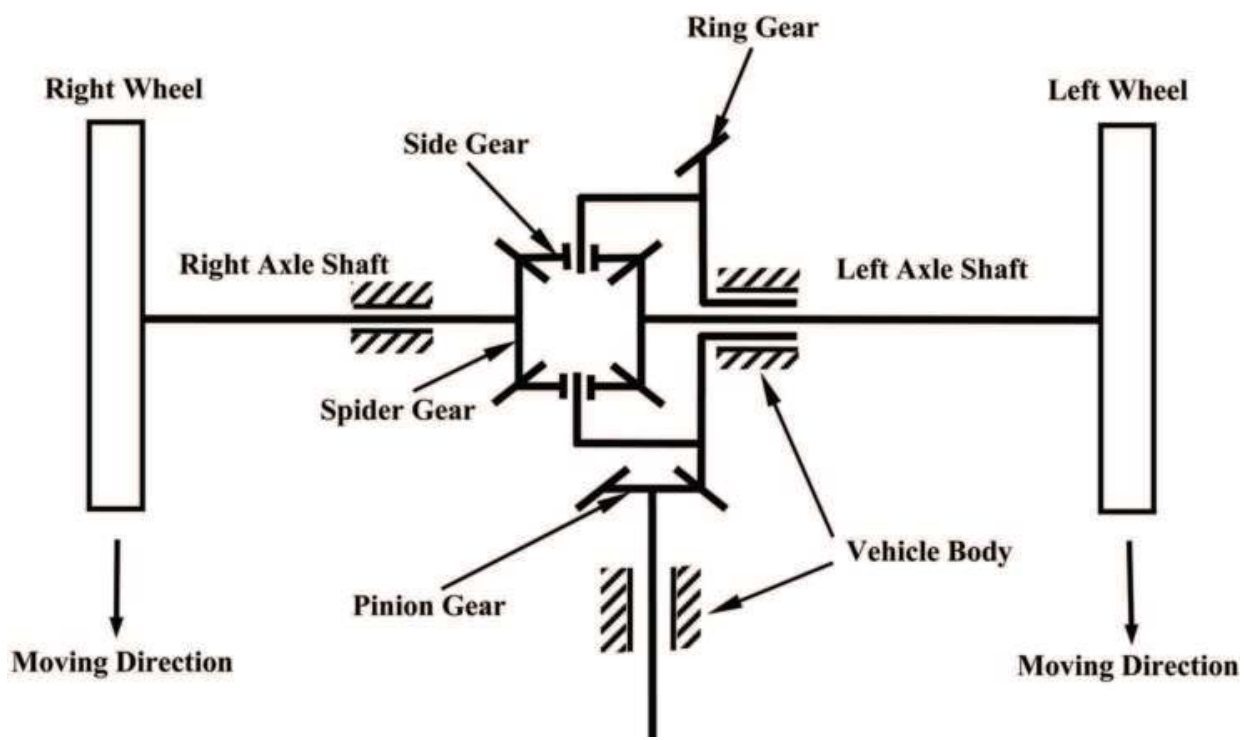

Fig. 3 the differential gear train mechanism

Another facility usually used in civil engineering is the inertial rammer shown in Fig. 4. This can also be modelled with a planar mechanism shown in Fig. 5. The apparent actuation is provided by the eccentric force of the eccentric rotor under the actuation of the electric motor. However, the motion of the rammer's body is indeterminate if the control of $F_{h}$ is not exerted to the handle. Therefore, the inertia rammer is not an underactuated mechanical system but a fully actuated system although the apparent actuation seems to be restricted to the eccentric force resulting from the eccentric rotating rotor.

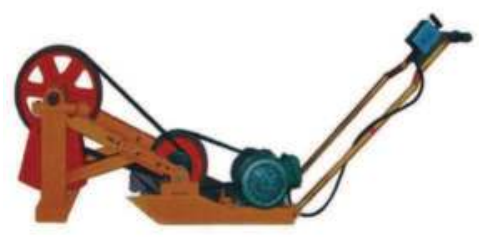

Fig. 4 the inertia rammer

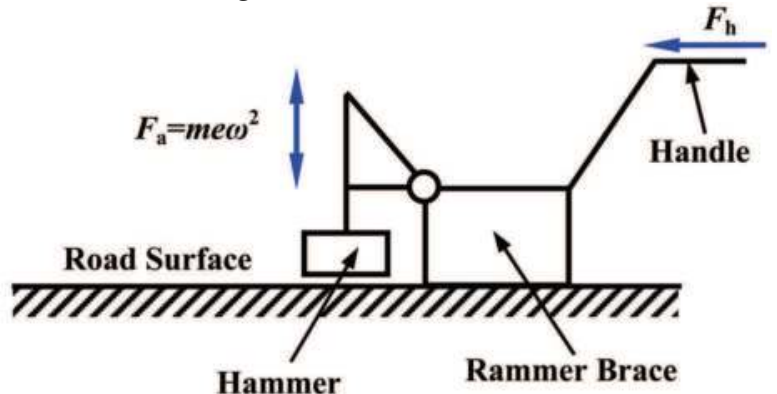

Fig. 5 the mechanism of the inertia rammer

From the above analysis, it is not difficult to find that all the underactuated mechanical systems are substantially actuation determinate from the viewpoint of mechanisms. 


\subsection{The essence of the over actuated manipulator}

Over-determinate actuation manipulators also witnesses wide applications in mechanical engineering, especially in biomechanical engineering. In order to investigate the essence of these manipulators, this section addresses this problem via some mechanism examples.

As a simple example, one might first investigate the motion of a vehicle with one degree of freedom under the actions of two persons shown in Fig. 6. The vehicle can only translate forward and backward along the road direction. However, two different actions are exerted to both sides of the vehicle. So, it is an over actuated mechanical system.

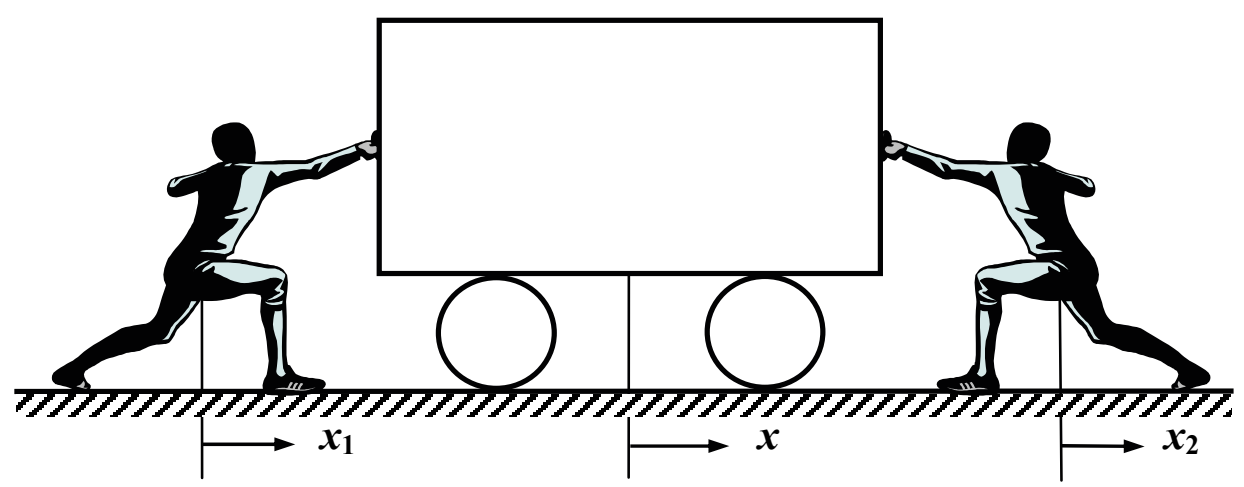

Fig. 6 an over actuated mechanical system

Out of question, the vehicle shown in Fig. 6 will move along the direction of the resultant force of the two persons, in spite of which the two actuations are not independent because these two actuations should satisfy that $x_{1}=x_{2}=x$. Otherwise, the two actuations might not do continuous work to the vehicle. These additional constraints are also called compliant equations.

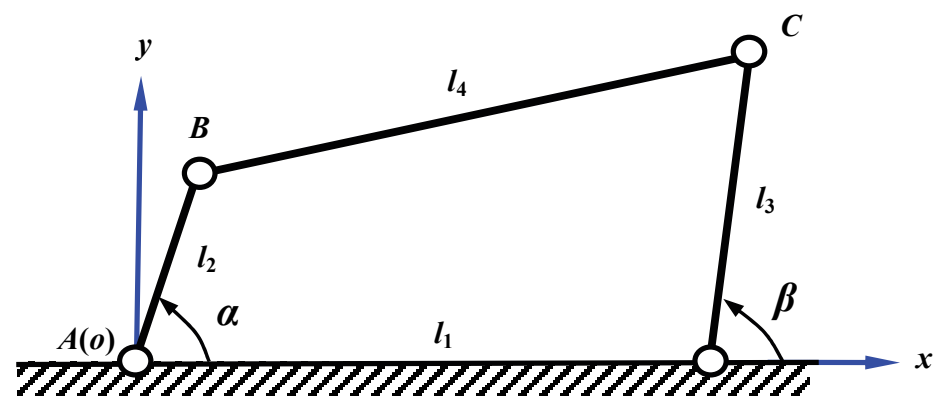

D

Fig. 7 a planar four-bar mechanism with two actuations

Next, one can consider a planar four-bar mechanism under two actuations shown in figure 7. Obviously, only one actuation is needed to control the mechanism. In engineering applications, however, it is also available to exert two actuations to increase the input torque or force to drive the mechanism to output a larger power. Therefore, the mechanism in such a case is a representative of the over actuated mechanical systems. 
Following, one can investigate the problems that might be ignored or misunderstood. For the sake of conveniences, a coordinate system is established by setting the origin to superimpose with revolute joint $A$ and $x$-axis along the link $A D$ and $y$-axis perpendicular upward to link $A D$. If the planar four-bar mechanism has a determinate motion, the equation below should hold:

$$
\left(l_{3} \cos \beta+l_{1}-l_{2} \cos \alpha\right)^{2}+\left(l_{3} \sin \beta-l_{2} \sin \alpha\right)^{2}=l_{4}^{2}
$$

Therefore, differentiating equation (27) with respect to time and rearranging yields:

$$
\frac{\dot{\beta}}{\dot{\alpha}}=\frac{l_{2}\left[l_{1} \sin \alpha+l_{3} \sin (\alpha-\beta)\right]}{l_{3}\left[l_{1} \sin \beta+l_{2} \sin (\alpha-\beta)\right]}
$$

where $\dot{\alpha}$ and $\dot{\beta}$ represent the angular velocities of the crank $A B$ and the rocker $D C$ shown in Fig. 7, individually.

Therefore, the actuations exerted to the crank $A B$ and the rocker $D C$ should keep in a precise relationship specified by equation (27). Otherwise, the link $B C$ might be cracked due to the increasing internal forces. Equation (27) or (28) is the compliant equation for the over actuated manipulator shown in Fig. 7.

Consequently, it is not difficult to find that there always are compliant constraint equations for the over actuated mechanical systems. And therefore, these mechanical systems are also substantially equally actuated.

\subsection{The problems to be noted in engineering applications}

The dexterity of an underactuated manipulator differs from the dexterity of a fully actuated one, even if their mechanical structures are identical. Therefore, the underactuated mechanical systems are widely used in the cases for fault tolerance and energy saving purposes. From the above analysis, one knows that any mechanical system that has a determinate motion should be an equally actuated system in essence. Next, one investigates an underactuated mechanical finger with return actuation shown in Fig. 8.

This mechanism is used in the finger of the United States patent initially applied by Gosselin et al (Gosselin \& Laliberté, 1998) for dexterity hand in 1998. The primary structure of the mechanism shown in Fig. 8 (Birglen \& Gosselin, 2006a) is a planar four-bar mechanism. Links $A B$ and $A D$ are simultaneously pivoted with the fixed wrist. Links $A B$ and $B C$ are connected by a passive spring. Next, the mobility of the mechanism will be investigated in several cases.

Firstly, when the finger does not contact any object, the links $A B$ and $B C$ connected by a passive spring might be disposed as one link, and therefore, $A B C D E$ forms one link and rotates about the fixed pivot, $A$, under the actuation of the force $F$. When $A B$ contacts a target object, the link $A B$ will degenerate to an unmovable base attached to the wrist, and therefore, the spring will be deform under the action of the force $F$ and finger $A B C D E$ forms a real four-bar mechanism. This will be holding until the side $B E$ also touches the boundary of the target object, after which the continuous increasing of the force $F$ will only results the deforming of the target object. 

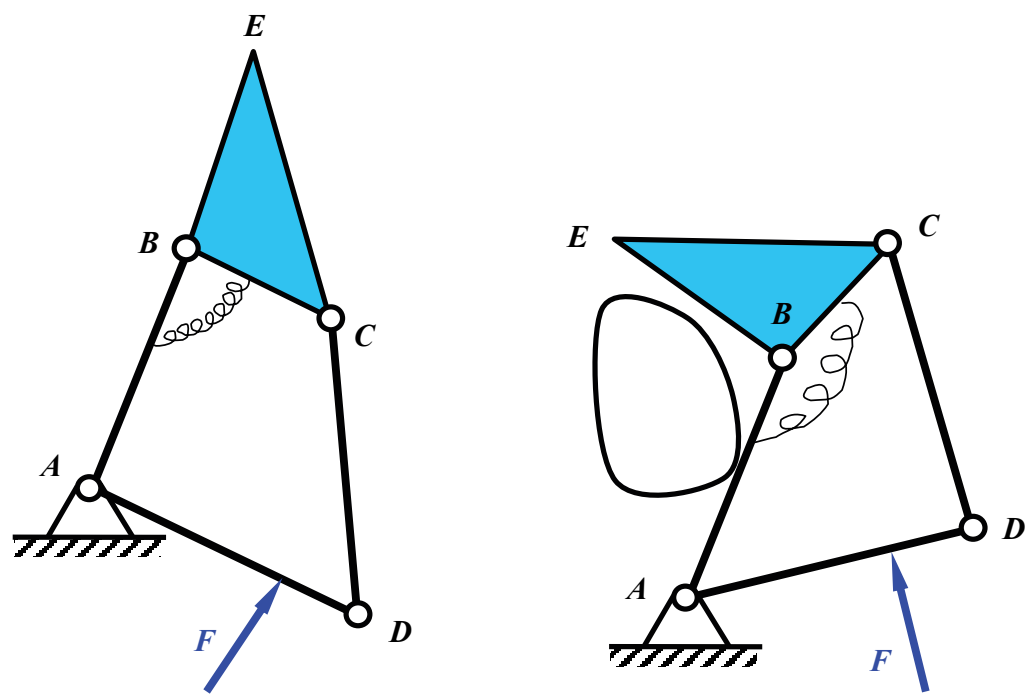

Fig. 8 underactuated mechanical finger with return actuation

The above analysis indicates that the so called underactuated mechanical finger is equally or fully actuated at any instant from the viewpoint of mechanisms. Consequently, no matter what kind does a mechanism belong to, it should have a determinate motion and equal actuation(s) at any instant, which should be particularly noticed in the concept design of underactuated mechanical systems. Theoretical and example analysis indicate that the underactuated, over actuated and fully actuated mechanical systems are all substantially equally actuated mechanisms.

\section{Synthesis of a spatial parallel manipulator with a specified mobility}

Usually, suspension is a general term of the equipments transforming forces and moments from the wheel to the vehicle body. Its primary function is to determine the geometry of the wheel motion during jounce and rebound, and to withstand forces and moments on the suspension in accelerating motion (Raghavan, 1996). The ride and handling characteristics of a vehicle are heavily dependent on the kinematic and compliance properties of the suspension mechanism (Raghavan, 2005). Compared with dependent suspensions, independent suspensions can eliminate undesirable dynamic phenomena such as shimmy and caster wobble resulting from wheel coupling in solid-axle suspensions (Raghavan, 1996). The most common independent suspension mechanisms utilized in automobiles are short-long arm suspension (Suh, 1998), the MacPherson strut (Raghavan, 2005), the multilink suspensions (Simionescu, 2002), and the short-long arm front suspension with a true kingpin (Murakami, 1989), etc. Most automotive independent suspension mechanisms are single degree-of-freedom mechanisms with the predominant motion being wheel jounce and rebound. In order to allow the wheel to pass the uneven terrain without slipping, Chakraborty and Ghosal (Chakraborty \& Ghosal, 2004) investigated the kinematics of a wheeled mobile robot moving on uneven terrain by modeling the wheels as a torus and proposing a lateral passive joint. Applications indicate that the wheel orientation and 
position parameters such as kingpin, caster, camber, toe change, axes distance, and the wheel track are primary consideration in the design of suspension mechanism. These parameters, as a matter of fact, are dependent on the wheel jounce and rebound, an independent parameter (Raghavan, 2005).

Therefore, a particular rigid guidance mechanism whose end-effector only has one straight line translation should maintain the orientation and position parameters invariable. Yan and Kuo (Yan \& Kuo, 2006) addressed the topological representations and characteristics of variable kinematic joints, which might be utilized in spatial mechanism synthesis. By considering workspace, dexterity, stiffness and singularity avoidance, Arsenault and Boudreau (Arsenault \& Boudreau, 2006) discussed the synthesis problems of planar parallel mechanisms. In the history of mechanism synthesis, a significant example is that the creation of linkages to produce exact straight line motion was an important engineering as well as a mathematical problem of the 19th century (Kempe, 1877). While many engineers and mathematicians were searching for a 4- 5- or 6-bar straight line linkage all suffered from the fact that they could not attain such a motion in the middle of 19th century, Peaucellier investigated an eight bar linkage shown in Fig. 9 and discovered he could generate an exact straight line motion from a rotary input.

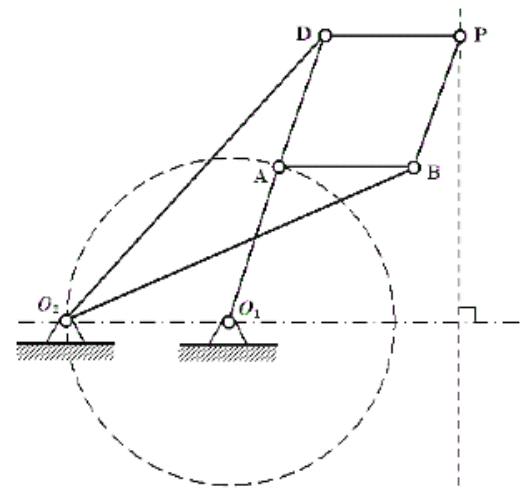

Fig. 9 Structure of Peaucellier-Lipkin Eight-Bar Linkage

This invention was recognized by several mathematicians as being very important to the design of general mathematical calculators (Kempe, 1877). This eight-link linkage was the one of the first to produce exact straight line motion and was independently invented by a French engineer named Peaucellier and by a Russian mathematician named Lipkin (Kempe, 1877), which is therefore often called Peaucellier-Lipkin eight-link linkage.

However, Peaucellier-Lipkin linkage is mostly utilized as a motion generator but not a rigid guidance mechanism. Obviously, because of its complexity, such a mechanism can not be used as a suspension in spite of the fact that it can really make the wheel move in a straight line during jounce and rebound. Therefore, this section first discusses the synthesis processes with the analytical model of the instantaneous mobility of a manipulator for the rigid guidance mechanism with the specified mobility; and then presents a rectilinear motion generating manipulator that can be utilized as a suspension mechanism.

The general synthesis process might be:

Step 1: Express the free motions required for the prescribed end-effector in Plücker coordinates at a Cartesian coordinate system. 
The Plücker coordinates of the specified motions should be firstly expressed in a Cartesian coordinate system. This chapter supposes that the twists of the free motion(s) of the endeffector are denoted by $\$_{E n d}^{F}$.

Step 2: Solve the constraint(s) exerted to the end-effector by its kinematic chain(s).

According to reciprocities between free motion(s) and constraint(s) of an end-effector, the constraint(s) applied to the end-effector can be solved with the equation (16):

$$
\left(\boldsymbol{\$}_{\text {End }}^{F}\right)^{T} E \boldsymbol{\$}_{\text {End }}^{C}=0
$$

where $\$_{E n d}^{F}$ indicates the specified free motion(s) of the end-effector and $\$_{\text {End }}^{C}$ denotes any constraint applied to the end-effector.

Step 3: Decide the number of kinematic chains, $m(m \geq 1)$, that will be used to connect the end-effector with the fixed base.

If every link in the chain is connected to at least two other links, the chain forms one or more closed loops and is called a closed kinematic chain; if not, the chain is referred to as open (Shigley \& Uicker, 1980). For the later open chain case, the synthesis is simply stated as: any kinematic chain is feasible if the twist basis, $\boldsymbol{\$}_{B_{i}}^{F}$, of the chain contains $\boldsymbol{\$}_{E n d}^{F}$. However, the following steps should be further discussed if the mechanism is a closed one.

Step 4: Synthesize the terminal constraint(s) of each kinematic chain.

Suppose

$$
\boldsymbol{\$}_{\text {End }}^{C}=\left[\begin{array}{llll}
\boldsymbol{\$}_{\text {End }}^{C_{1}} & \boldsymbol{\$}_{\text {End }}^{C_{2}} & \cdots & \boldsymbol{\$}_{\text {End }}^{C_{n}}
\end{array}\right]
$$

where $n$ indicates the dimension of the constraint basis of the end-effector.

Suppose that the terminal constraint(s) of the $i$ th $(i=1,2, \cdots, m)$ kinematic chain is denoted by $\$_{i}^{C}$, the terminal constraint(s) of the chain might be synthesized with:

$$
\$_{i}^{C}=\$_{E n d}^{C} K_{i}
$$

where $K_{i}=\left[\begin{array}{llll}K_{i 1} & K_{i 2} & \cdots & K_{i n_{i}}\end{array}\right]$, and $K_{i j}=\left(\begin{array}{llll}k_{i 1} & k_{i 2} & \cdots & k_{i n}\end{array}\right)^{T}$ and $j=1,2, \cdots, n_{i}$.

For a feasible mechanism that makes the end-effector only have the prescribed free motion(s), the necessary and sufficient criterion is that the resultant terminal constraint(s) of all these $m$ kinematic chain(s), $\bigcup_{i=1}^{m} \$_{i}^{C}$, should be equivalent to $\$_{E n d}^{C}$. This is called the construction criterion 1 of the feasible kinematic chains.

The necessity and sufficiency of this criterion can be immediately deduced from equation (29) with linear algebra theory.

Step 5: Solve the twist basis of the $i$ th kinematic chain with the terminal constraints, $\$_{i}^{C}$, synthesized in step 4 . 
With reciprocal screw theory, a basis of the twist(s) of the ith kinematic chain, denoted by $\$_{B_{i}}^{F}$, can be obtained by solving the following equation:

$$
\left(\boldsymbol{\$}_{i}^{C}\right)^{T} E \$_{B_{i}}^{F}=0
$$

where $\$_{i}^{C}$ represents the terminal constraint(s) of the $i$ th kinematic chain synthesized in step 4.

Step 6: Synthesize the twist(s) of the $i$ th kinematic chain with the twist basis of the ith chain, $\$_{B_{i}}^{F}$, obtained in step 5 .

Suppose

$$
\boldsymbol{\$}_{B_{i}}^{F}=\left[\begin{array}{llll}
\boldsymbol{S}_{B_{i}}^{F_{1}} & \boldsymbol{\$}_{B_{i}}^{F_{2}} & \cdots & \boldsymbol{\$}_{B_{i}}^{F_{n_{i}}}
\end{array}\right]
$$

where $n_{i}$ indicates the dimension of the twist basis of the $i$ th $(i=1,2, \cdots, m)$ kinematic chain. According to linear algebra, any twist of the $i$ th kinematic chain can be expressed as the linear combinations of the twist basis of the chain:

$$
\$_{i}^{F_{a}}=\$_{B_{i}}^{F} C
$$

where $C=\left(\begin{array}{llll}c_{1} & c_{2} & \cdots & c_{n_{i}}\end{array}\right)^{T}$.

Consequently, the twists of each kinematic chain can be synthesized through equation (34). However, in order to keep the twists of the ith chain to be equivalent to the twist basis of the chain, the rank of the total twists synthesized through equation (34) should equal the dimension of the twist basis of the chain. This is called the construction criterion 2 of the feasible kinematic chains.

The necessary and sufficient of this criterion can be immediately obtained from equation (32).

According to the construction criteria 1 and 2, the required synthesis target of a mechanism can be gradually accomplished with the above six steps. Obviously, with these six steps, different person might synthesize different kinematic chains and different mechanisms. However, all the end-effectors of the mechanisms synthesized with the same criteria will surely have the identical specified free motion(s).

The next section will apply these steps to synthesize a rigid guidance mechanism that can be utilized as a suspension of an automobile.

The synthesis target now is to use the least number of links and pure revolute joints to design a mechanism whose end-effector has one pure translation along an exact straight line; therefore, the mechanism must be a closed one. The reason is that it will need at least two actuations to generate a pure straight line translation with an open chain mechanism. And therefore, for the purpose of the suspension required, one at least needs two kinematic chains to generate a pure straight line translation with one actuation input. According to step 1, the specified free motion of the end-effector should be expressed in a Cartesian 
coordinate system. Without loss of generality, the precise straight line translation of the endeffector can be assumed to parallel $z$-axis. Therefore, the free motion can be described in Plücker coordinates as:

$$
\$_{\text {End }}^{F}=\left(\begin{array}{llllll}
0 & 0 & 0 & 0 & 0 & 1
\end{array}\right)^{T}
$$

So, the target now can be depicted as whether one can find two sets of screws whose pitches represented by equation (3) are all zeros provided that they were all reciprocal to $\$_{E n d}^{F}$ of equation (35).

According to step 2, substituting equation (35) into equation (29) yields the constraints exerted to the end-effector, $\$_{E n d}^{C}$ :

$$
\boldsymbol{\$}_{\text {End }}^{C}=\left[\begin{array}{lllll}
\boldsymbol{\$}_{\text {End }}^{C_{1}} & \boldsymbol{\$}_{\text {End }}^{C_{2}} & \boldsymbol{\$}_{\text {End }}^{C_{3}} & \boldsymbol{\$}_{\text {End }}^{C_{4}} & \boldsymbol{\$}_{\text {End }}^{C_{5}}
\end{array}\right]
$$

where $\quad \$_{E n d}^{C_{1}}=\left(\begin{array}{llllll}1 & 0 & 0 & 0 & 0 & 0\end{array}\right)^{T} \quad$ represents a force along $x$-axis, $\boldsymbol{\$}_{\text {End }}^{C_{2}}=\left(\begin{array}{llllll}0 & 1 & 0 & 0 & 0 & 0\end{array}\right)^{T}$ represents a force along $y$-axis, $\boldsymbol{\$}_{\text {End }}^{C_{3}}=\left(\begin{array}{llllll}0 & 0 & 0 & 1 & 0 & 0\end{array}\right)^{T}$ represents a torque about $x$-axis, $\$_{\text {End }}^{C_{4}}=\left(\begin{array}{llllll}0 & 0 & 0 & 0 & 1 & 0\end{array}\right)^{T}$ represents a torque about $y$ axis, and $\$_{E n d}^{C_{5}}=\left(\begin{array}{llllll}0 & 0 & 0 & 0 & 0 & 1\end{array}\right)^{T}$ represents a torque about $z$-axis.

From equation (32), it is not difficult to find that the sum of the number of the independent twists and the number of the terminal constraints of a chain is six. In order to reduce the number of revolute joints, one might have to increase the number of the terminal constraints of the chains as many as possible. According to equations (31) and (36), the maximum number of the terminal constraints of a chain is five. However, if such a structure scheme is used, one may find each kinematic chain only consists of one revolute joint, which is unfeasible in reality. Similarly, it is not difficult to find that only when each kinematic chain provides three terminal constraints at most, can the structure scheme is feasible.

With equation (31), one can synthesize the terminal constraints of these two kinematic chains, individually. Selecting different $k_{i}(i=1,2, \cdots, 5)$ and substituting them into equation (31), one can synthesize three independent terminal constraints for the first kinematic chain, for example:

Assuming $K_{1}=\left[\begin{array}{lll}1 & 0 & 0 \\ 0 & 0 & 0 \\ 0 & 0 & 0 \\ 0 & 1 & 0 \\ 0 & 0 & 1\end{array}\right]$, one obtains

$$
\$_{1}^{C}=\left[\begin{array}{lll}
\$_{1}^{C_{1}} & \$_{1}^{C_{2}} & \$_{1}^{C_{3}}
\end{array}\right]
$$


where $\$_{1}^{C_{1}}=\left(\begin{array}{llllll}1 & 0 & 0 & 0 & 0 & 0\end{array}\right)^{T}$ indicates a force along $x$-axis, $\$_{1}^{C_{2}}=\left(\begin{array}{llllll}0 & 0 & 0 & 0 & 1 & 0\end{array}\right)^{T}$ indicates a torque about $y$-axis, and $\$_{1}^{C_{3}}=\left(\begin{array}{llllll}0 & 0 & 0 & 0 & 0 & 1\end{array}\right)^{T}$ indicates a torque about $z$ axis.

Assuming $K_{2}=\left[\begin{array}{ccc}a & 0 & 0 \\ b & 0 & 0 \\ 0 & b & 0 \\ 0 & -a & 0 \\ 0 & 0 & 1\end{array}\right]$, one can obtain

$$
\$_{2}^{C}=\left[\begin{array}{lll}
\$_{2}^{C_{1}} & \$_{2}^{C_{2}} & \$_{2}^{C_{3}}
\end{array}\right]
$$

where $\$_{1}^{C_{1}}=\left(\begin{array}{llllll}a & b & 0 & 0 & 0 & 0\end{array}\right)^{T}$ denotes a force along the direction $\left(\begin{array}{lll}a & b & 0\end{array}\right)^{T}$, $\$_{1}^{C_{2}}=\left(\begin{array}{llllll}0 & 0 & 0 & b & -a & 0\end{array}\right)^{T}$ denotes a torque about the direction $\left(\begin{array}{lll}b & -a & 0\end{array}\right)^{T}$, $\$_{1}^{C_{3}}=\left(\begin{array}{llllll}0 & 0 & 0 & 0 & 0 & 1\end{array}\right)^{T}$ denotes a torque about $z$-axis and $a b \neq 0$.

Because dimspan $\left\{K_{1}, K_{2}\right\}=5$, the resultant terminal constraints of these 2 kinematic chains, $\bigcup_{i=1}^{2} \$_{i}^{C}$ must be equivalent to $\$_{E n d}^{C}$. So the construction criterion 1 is satisfied.

According to equation (32), one immediately obtains the twist bases for the two kinematic chains with equations (37) and (38):

$$
\boldsymbol{\$}_{B_{1}}^{F}=\left[\begin{array}{lll}
\boldsymbol{\$}_{B_{1}}^{F_{1}} & \boldsymbol{\$}_{B_{1}}^{F_{2}} & \boldsymbol{\$}_{B_{1}}^{F_{3}}
\end{array}\right]
$$

where $\quad \$_{B_{1}}^{F_{1}}=\left(\begin{array}{lllllll}1 & 0 & 0 & 0 & 0 & 0\end{array}\right)^{T} \quad$ represents a rotation about $x$-axis, $\boldsymbol{\$}_{B_{1}}^{F_{2}}=\left(\begin{array}{llllll}0 & 0 & 0 & 0 & 1 & 0\end{array}\right)^{T}$ represents a translation along $y$-axis, $\boldsymbol{\$}_{B_{1}}^{F_{3}}=\left(\begin{array}{llllll}0 & 0 & 0 & 0 & 0 & 1\end{array}\right)^{T}$ represents a translation along $z$-axis, and

$$
\$_{B_{2}}^{F}=\left[\begin{array}{lll}
\$_{B_{2}}^{F_{1}} & \$_{B_{2}}^{F_{2}} & \$_{B_{2}}^{F_{3}}
\end{array}\right]
$$

where $\$_{B_{2}}^{F_{1}}=\left(\begin{array}{llllll}\cos \alpha & \sin \alpha & 0 & 0 & 0 & 0\end{array}\right)^{T}$ denotes a rotation about the direction $\left(\begin{array}{lll}\cos \alpha & \sin \alpha & 0\end{array}\right)^{T}, \quad \$_{B_{2}}^{F_{2}}=\left(\begin{array}{llllll}0 & 0 & 0 & -\sin \alpha & \cos \alpha & 0\end{array}\right)^{T}$ denotes a translation along the direction $\left(\begin{array}{lll}-\sin \alpha & \cos \alpha & 0\end{array}\right)^{T}, \$_{B_{2}}^{F_{3}}=\left(\begin{array}{llllll}0 & 0 & 0 & 0 & 0 & 1\end{array}\right)^{T}$ denotes a translation along $z$-axis, and $\cos \alpha=\frac{a}{\sqrt{a^{2}+b^{2}}}$ and $\sin \alpha=\frac{b}{\sqrt{a^{2}+b^{2}}}$.

According to step 6, one can synthesize the twists of the two kinematic chains with their twist bases (39) and (40), individually. Considering the construction criterion 2, one can find 
that the least number of twists in each kinematic chain is three. Therefore, the twist of the first kinematic chain can be synthesized below with equation (34):

$$
\$_{1}^{F a}=c_{1} \$_{B_{1}}^{F_{1}}+c_{2} \boldsymbol{\$}_{B_{1}}^{F_{2}}+c_{3} \$_{B_{1}}^{F_{3}}=\left(\begin{array}{llllll}
c_{1} & 0 & 0 & 0 & c_{2} & c_{3}
\end{array}\right)^{T}
$$

Substituting equation (41) into equation (3) yields:

$$
h_{1}^{F a}=0
$$

Equation (42) indicates that any twist having the form of equation (41) will naturally satisfy the free motion requirements of the end-effector. The Cartesian coordinates of the joint, $\boldsymbol{r}_{A_{1}}$, can be found from equations (7) and (9):

$$
\boldsymbol{r}_{A_{1}}=\frac{\boldsymbol{s} \times \boldsymbol{s}_{0}}{\|\boldsymbol{s}\|^{2}}+\frac{a \boldsymbol{s}}{\|\boldsymbol{s}\|}=\left(\begin{array}{lll}
a & -\frac{c_{3}}{c_{1}} & \frac{c_{2}}{c_{1}}
\end{array}\right)^{T}
$$

To make the twists of the chain be equivalent to the twist basis, there are at least three twists indicated in the form of equation (41).

Suppose $c_{1}=1$ and the three joints' coordinates are

$$
\left\{\begin{array}{l}
\boldsymbol{r}_{A}=\left(\begin{array}{lll}
a & 0 & 0
\end{array}\right)^{T} \\
\boldsymbol{r}_{B}=\left(\begin{array}{lll}
a & y_{B} & z_{B}
\end{array}\right)^{T} \\
\boldsymbol{r}_{C}=\left(\begin{array}{lll}
a & y_{C} & z_{C}
\end{array}\right)^{T}
\end{array}\right.
$$

then, the twists of the first kinematic chain will be:

$$
\$_{A B C}=\left[\begin{array}{lll}
\$_{1}^{F_{A}} & \$_{1}^{F_{B}} & \$_{1}^{F_{C}}
\end{array}\right]
$$

where $\quad \$_{1}^{F_{A}}=\left(\begin{array}{llllll}1 & 0 & 0 & 0 & 0 & 0\end{array}\right)^{T} \quad$ represents a rotation about $x$-axis, $\$_{1}^{F_{B}}=\left(\begin{array}{lllllll}1 & 0 & 0 & 0 & z_{B} & -y_{B}\end{array}\right)^{T}$ represents a rotation about a line passing through point $\left(\begin{array}{lll}x_{B} & y_{B} & z_{B}\end{array}\right)$ and paralleling $x$-axis, and $\$_{1}^{F_{C}}=\left(\begin{array}{llllll}1 & 0 & 0 & 0 & z_{C} & -y_{C}\end{array}\right)^{T}$ represents a rotation about a line passing through point $\left(\begin{array}{llll}x_{C} & y_{C} & z_{C}\end{array}\right)$ and paralleling $x$-axis.

According to equation (34), a twist of the second kinematic chain, denoted by $\$_{2}^{F a}$, can be expressed as:

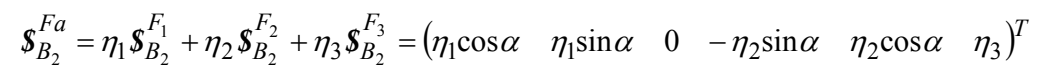

where $\eta_{i}$ denote real numbers and $i=1,2,3$.

Substituting equation (44) into equation (3) yields:

$$
h_{2}^{F a}=0
$$


Equation (45) indicates that any twist having the form of equation (44) will naturally satisfy the free motion requirements of the end-effector.

The Cartesian coordinates of the joint, $\boldsymbol{r}_{A_{2}}$, can be found from equations (7) and (9):

$$
\boldsymbol{r}_{A_{2}}=\frac{\boldsymbol{s} \times \boldsymbol{s}_{0}}{\|\boldsymbol{s}\|^{2}}+\frac{b \boldsymbol{s}}{\|\boldsymbol{s}\|}=\left(\frac{\eta_{3}}{\eta_{1}} \sin \alpha+b \cos \alpha-\frac{\eta_{3}}{\eta_{1}} \cos \alpha+b \sin \alpha \quad \frac{\eta_{2}}{\eta_{1}}\right)^{T}
$$

To keep the twists of the chain be equivalent to the twist basis, one can only select three independent twists indicated with equation (44) by selecting three sets of $\left(\begin{array}{lll}\eta_{1} & \eta_{2} & \eta_{3}\end{array}\right)$.

If one supposes $\eta_{1}=1, \eta_{2}=z_{F}, \eta_{3}=x_{F} \sin \alpha-y_{F} \cos \alpha$ and $b=x_{F} \cos \alpha+y_{F} \sin \alpha$, he obtains the coordinates of revolute joint $F, \boldsymbol{r}_{F}=\left(\begin{array}{lll}x_{F} & y_{F} & z_{F}\end{array}\right)^{T}$; similarly, if one supposes $\eta_{1}=1$, $\eta_{2}=z_{E}, \quad \eta_{3}=x_{E} \sin \alpha-y_{E} \cos \alpha$ and $b=x_{E} \cos \alpha+y_{E} \sin \alpha$, he obtains the coordinates of revolute joint $E, \quad \boldsymbol{r}_{E}=\left(\begin{array}{lll}x_{E} & y_{E} & z_{E}\end{array}\right)^{T} ;$ and if one supposes $\eta_{1}=1, \eta_{2}=z_{D}$, $\eta_{3}=x_{D} \sin \alpha-y_{D} \cos \alpha$ and $b=x_{D} \cos \alpha+y_{D} \sin \alpha$, he can obtain the coordinates of revolute joint $D, \mathrm{r}_{D}=\left(\begin{array}{lll}x_{D} & y_{D} & z_{D}\end{array}\right)^{T}$. Therefore, the three joints' coordinates can be assumed

$$
\left\{\begin{array}{l}
\boldsymbol{r}_{F}=\left(\begin{array}{lll}
x_{F} & y_{F} & z_{F}
\end{array}\right)^{T} \\
\boldsymbol{r}_{E}=\left(\begin{array}{lll}
x_{E} & y_{E} & z_{E}
\end{array}\right)^{T} \\
\boldsymbol{r}_{D}=\left(\begin{array}{lll}
x_{D} & y_{D} & z_{D}
\end{array}\right)^{T}
\end{array}\right.
$$

then, the twists of the second kinematic chain will be:

$$
\$_{F E D}=\left[\begin{array}{lll}
\$_{2}^{F_{F}} & \boldsymbol{\$}_{2}^{F_{E}} & \$_{2}^{F_{D}}
\end{array}\right]
$$

where $\$_{2}^{F_{F}}=\left(\begin{array}{llllll}\cos \alpha & \sin \alpha & 0 & 0 & 0 & 0\end{array}\right)^{T}$ represents a rotation about a line passing through the origin of the coordinate system and in the direction $(\cos \alpha \sin \alpha \quad 0)^{T}$, $\$_{2}^{F_{E}}=\left(\begin{array}{llllll}\cos \alpha & \sin \alpha & 0 & -z_{E} \sin \alpha & z_{E} \cos \alpha & x_{E} \sin \alpha-y_{E} \cos \alpha\end{array}\right)^{T}$ represents a rotation about a line passing through point $\left(\begin{array}{lll}x_{E} & y_{E} & z_{E}\end{array}\right)$ and in the direction $(\cos \alpha \sin \alpha)^{T}$, and $\$_{2}^{F_{D}}=\left(\begin{array}{llllll}\cos \alpha & \sin \alpha & 0 & -z_{D} \sin \alpha & z_{D} \cos \alpha & x_{D} \sin \alpha-y_{D} \cos \alpha\end{array}\right)^{T}$ represents a rotation about a line passing through point $\left(\begin{array}{llll}x_{D} & y_{D} & z_{D}\end{array}\right)$ and in the direction $\left(\begin{array}{lll}\cos \alpha & \sin \alpha & 0\end{array}\right)^{T}$.

With equations (43) and (46), one can synthesize a spatial six link mechanism $A B C D E F$ shown in Fig. 10. It is not difficult to find that $\alpha$ is the angle from $x$-axis to the $y^{\prime}$-axis of the revolute joint $F$ and the revolute joints $F, E$ and $D$ have the same axis direction, which is denoted by $\boldsymbol{n}_{F E D}=\left(\begin{array}{lll}\cos \alpha & \sin \alpha & 0\end{array}\right)^{T}$. 


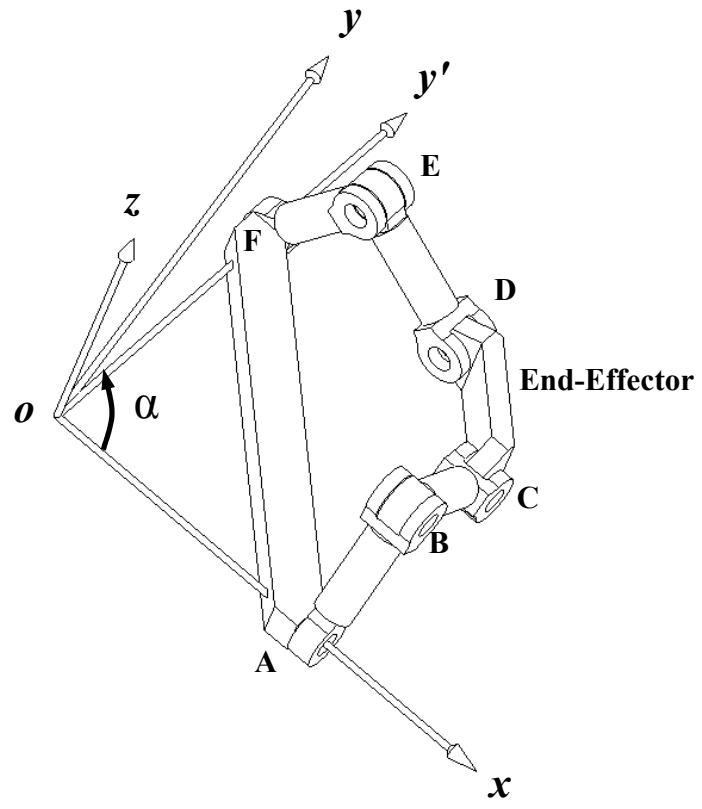

Fig. 10 a Spatial Six-Link Mechanism with a Straight line Translational End-Effector

From the above analysis, it is not difficult to find that the two kinematic chains $A B C$ and $F E D$ can surely guarantee the pure straight line translation of the end-effector $C D$ so long as $\$_{A B C}$ and $\$_{F E D}$ do not descend in ranks. To analyze the sensitivity of the structure stability to the angle $\alpha$, one should turn to the equations (37) and (38) and investigate the resultant terminal constraints, which can be expressed with:

$$
\$_{C D}^{C}(\alpha)=\left[\begin{array}{ccccc}
1 & \cos \alpha & 0 & 0 & 0 \\
0 & \sin \alpha & 0 & 0 & 0 \\
0 & 0 & 0 & 0 & 0 \\
0 & 0 & \sin \alpha & 0 & 0 \\
0 & 0 & -\cos \alpha & 1 & 0 \\
0 & 0 & 0 & 0 & 1
\end{array}\right]
$$

where $\cos \alpha=\frac{a}{\sqrt{a^{2}+b^{2}}}$ and $\sin \alpha=\frac{b}{\sqrt{a^{2}+b^{2}}}$.

If the terminal constraints denoted by $\$_{C D}^{C}(\alpha)$ are well conditioned, the mechanism will have fine structure stability. From equations (29) and (47), one can find that the end-effector will have one straight line translation along $z$-axis so long as $\operatorname{rank}\left(\$_{C D}^{C}(\alpha)\right)=5$, which can be immediately transformed to investigate the following sub matrix of $\$_{C D}^{C}(\alpha)$ : 


$$
A(\alpha)=\left[\begin{array}{ccccc}
1 & \cos \alpha & 0 & 0 & 0 \\
0 & \sin \alpha & 0 & 0 & 0 \\
0 & 0 & \sin \alpha & 0 & 0 \\
0 & 0 & -\cos \alpha & 1 & 0 \\
0 & 0 & 0 & 0 & 1
\end{array}\right]
$$

Letting $\operatorname{det}(A(\alpha))=0$, one immediately obtains $\alpha=0$ or $\alpha=\pi$. Therefore, in order to keep the end-effector $C D$ have one straight line translation along $z$-axis, there will be $\alpha \neq 0$ and $\alpha \neq \pi$. So, the rigid guidance mechanism synthesized in this chapter has a wider adaptation of angle between the planes of its two kinematic chains. Now, the sensitivity of the structure stability to the angle $\alpha$ of the mechanism can be judged by the condition number of matrix $A(\alpha)$ (Kelley, 1995). Let

$$
\operatorname{cond}(A)_{2}=\|A\|_{2}\left\|A^{-1}\right\|_{2}=\sqrt{\frac{\lambda_{\max }\left(A^{T} A\right)}{\lambda_{\min }\left(A^{T} A\right)}}=1
$$

where $\operatorname{cond}(A)_{2}$ indicates the condition number of matrix $A,\|A\|_{2}$ indicates the 2-norm of matrix $A$, and $\lambda\left(A^{T} A\right)$ indicates the eigenvalues of matrix $A^{T} A$.

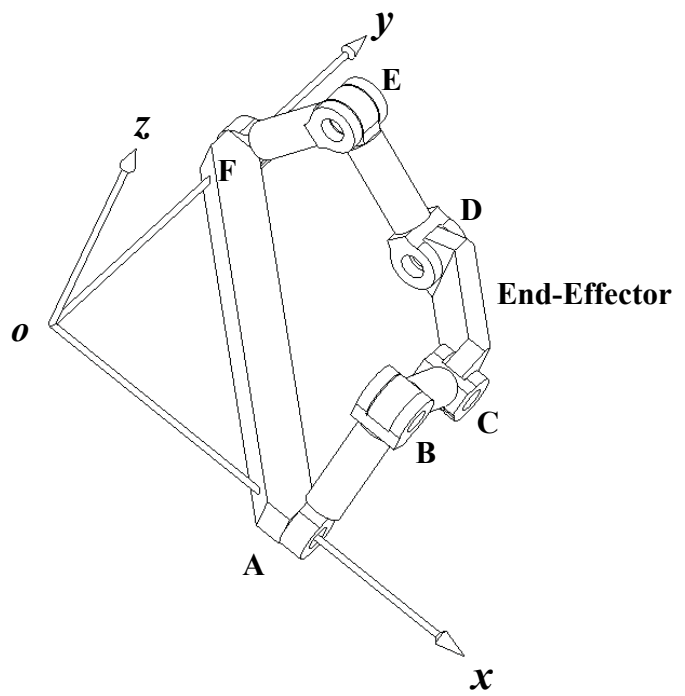

Fig. 11 a Spatial Six-Link Mechanism with the Best Structure Stability The solution of equation (48) is:

$$
\alpha=\frac{\pi}{2}
$$


Equation (49) indicates that the mechanism will have the best structure stability when $\alpha=\frac{\pi}{2}$, which is shown in Fig. 11. Compared with Peaucellier-Lipkin eight-link linkage, the spatial six-link mechanism synthesized in this chapter has the least links and revolute joints, and the whole end-effector $C D$ can make an exact straight line translation while PeaucellierLipkin eight-link linkage can only allow one specified point to make such a motion.

As a matter of fact, the mechanism shown in Fig. 11 is a Sarrus linkage. However, the mechanism proposed here does not necessarily require that the two kinematic chains must within two orthogonal planes which are needed for Sarrus linkage. The so-called Sarrus linkage, which is shown in Fig. 12, is a linkage that converts circular motion to linear motion by using hinged squares. The square end-effector $C_{1} C_{2}$ can make an exact straight line translation along $z$-axis which shows better properties both in mechanical structure and in kinematics than those of Peaucellier-Lipkin eight-link linkage. However, because of the limited workspace and the uneconomic mechanism architecture, the restrictions of Sarrus linkage shown in Fig. 12 compared with the one shown in Fig. 11 are obvious.

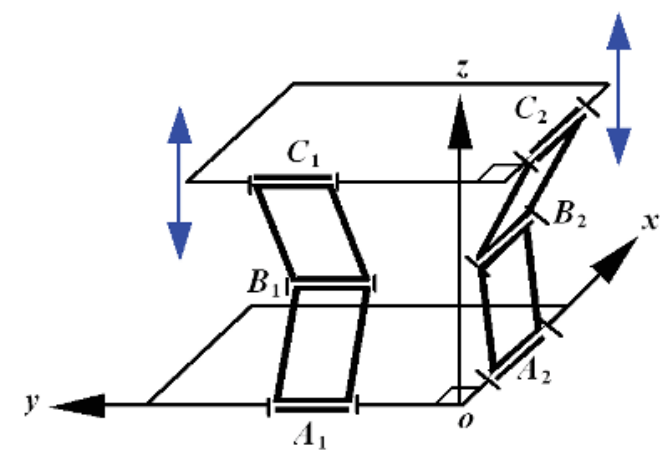

Fig. 12 the Structure of Sarrus Linkage

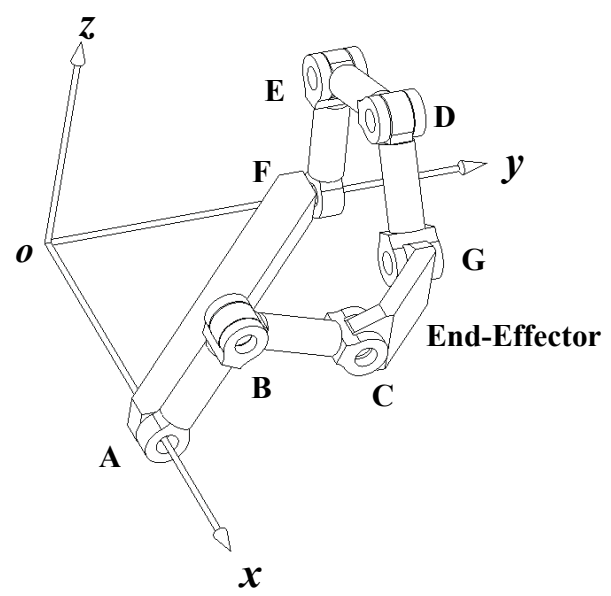

Fig. 13 Configuration of the Spatial Seven-Link Mechanism 
As mentioned in step 6, in order to keep the twists of the chain to be equivalent to the twist basis, the rank of the twists of each kinematic chain synthesized through equation (34) should equal the dimension of its twist basis. Therefore, if one or more such twists are added to each kinematic chain, the free motions of the end-effector will not be changed. As an example, the mechanism shown in Fig. 13 is the derivative form of that in Fig. 10 by adding one twist $\$_{G_{1}}$ to the kinematic chain FED. Where

$$
\$_{G}=\left(\begin{array}{llllll}
0 & 1 & 0 & -z_{G} & 0 & x_{G}
\end{array}\right)^{T}
$$

The two kinematic chains of the end-effector $C D$ are now changed to be $A B C$ and $F E G_{1} D$. The twists of them two are:

$$
\left\{\begin{array}{l}
\$_{A B C}=\left[\begin{array}{lll}
\$_{1}^{F_{A}} & \$_{1}^{F_{B}} & \$_{1}^{F_{C}}
\end{array}\right] \\
\$_{F E G D}=\left[\begin{array}{llll}
\$_{2}^{F_{F}} & \$_{2}^{F_{E}} & \$_{2}^{F_{G}} & \$_{2}^{F_{D}}
\end{array}\right]
\end{array}\right.
$$

It is not difficult to find that the terminal constraints of kinematic chains $A B C$ and $F E G_{1} D$ are still expressed by equation (36). And therefore, the free motion of the end-effector $C D$ is still a straight line translation along $z$-axis shown in Fig. 13. As a result, the free motions of the end-effector will not be changed if one or more revolute joints whose Plücker coordinates have the form of equation (44) are added in the second kinematic chain. Similarly, the free motions of the end-effector will not be changed either if one or more revolute joints whose Plücker coordinates have the form of equation (41) are added to the first kinematic chain.

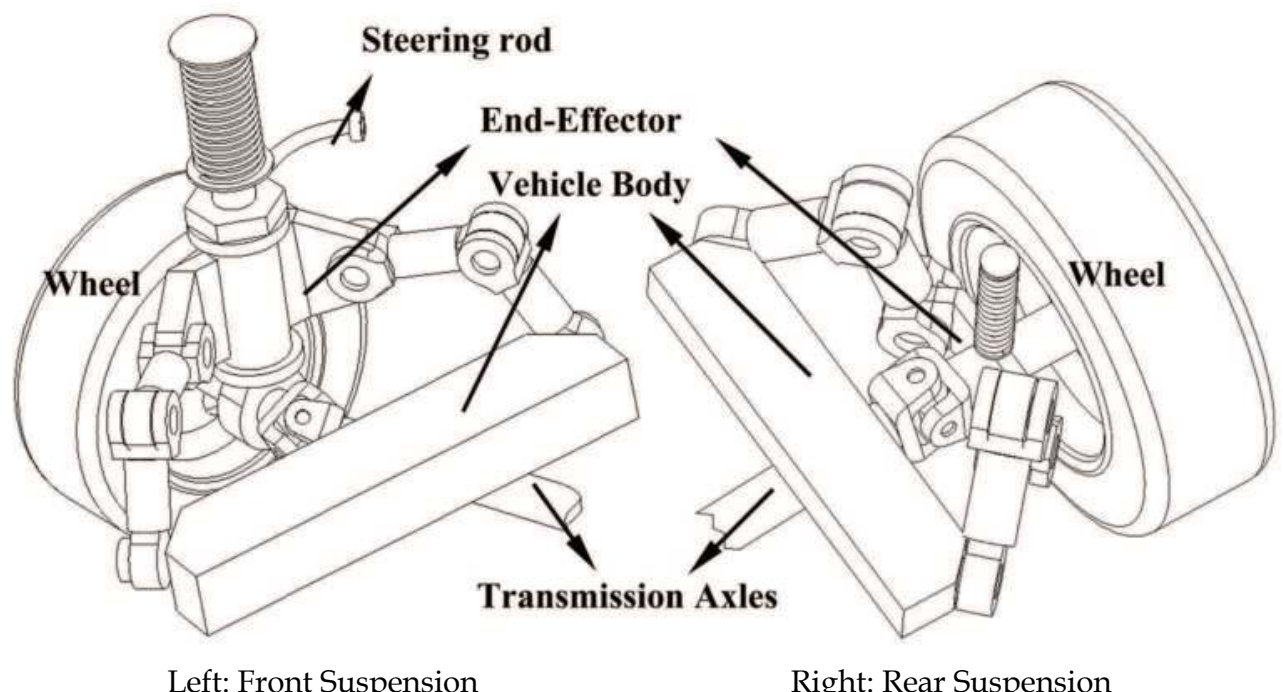

Fig. 14 a Front Suspension and a Rear Suspension 
For engineering applications, the end-effector $C D$ in Fig. 11 or Fig. 13 can be utilized as the guiding equipment of a mechanism that requires a precise linear translation, such as the independent suspension of automobile. Because the end-effector of the rigid guidance mechanism can make an exact straight line translation, the front and rear suspensions made up of such a mechanism shown in Fig. 14 allow the orientation and position parameters of the wheels such as kingpin, caster, camber, and axes distance and wheel track to be constant. These merits not only enhance the ride and handling of the vehicles, but also reduce the wearing of the tires during jounce and rebound.

\section{Conclusion}

This chapter focuses on the mobility analysis and synthesis of spatial parallel manipulators. It focuses on developing an analytical methodology to investigate the instantaneous DOF of the end-effector of a parallel manipulator and the instantaneous controllability of the endeffector from the viewpoint of the possible actuation schemes for the parallel manipulator. Via comparing the differences and essential mobility of a set of underactuated, over actuated and equally actuated manipulators, this chapter demonstrates that the underactuated, over actuated and fully actuated manipulators are all substantially equally actuated mechanisms. This work is significantly important for a designer to contrive his or her manipulators with underactuated or over actuated structures. Based on the analytical model of the DOF of a spatial parallel manipulator, this chapter also investigates a general process to synthesize the manipulators with specified mobility. The outstanding characteristics of the synthesis method are that the whole process is also analytical and each step can be programmed at a computer. Because of the restrictions of the traditional general mobility formulas for spatial mechanisms, a lot of mechanisms that might not be synthesized directly with the general mobility formulas could be synthesized with this analytical theory of degrees of freedom for spatial mechanisms.

\section{Acknowledgements}

This research was supported by FANEDD under Grant 200741 and the National Natural Science Foundation of China under Grant 50425516. The authors gratefully acknowledge these support agencies.

\section{References}

Arsenault, M. \& Boudreau, R. (2006). Synthesis of Planar Parallel Mechanisms While Considering Workspace, Dexterity, Stiffness and Singularity Avoidance, ASME Journal of Mechanical Design, Vol. 128, No. 1, (January, 2006)69-78 ISSN: 1050-0472

Ball, R. S. (First Published 1900, Reprinted 1998). a Treatise on the Theory of Screws, Cambridge University Press, ISBN 0-521-63650-7, Cambridge

Birglen, L. \& Gosselin, C. M. (2006a). Geometric Design of Three-Phalanx Underactuated Fingers, ASME Journal of Mechanical Design, Vol. 128, No. 2, (March 2006)356-364, ISSN: 1050-0472 
Birglen, L. \& Gosselin, C. M. (2006b). Grasp-state plane analysis of two-phalanx underactuated fingers, Mechanism and Machine Theory, Vol. 41, No. 7, (July 2006) 807-822, ISSN: 0094-114X

Chakraborty, N. \& Ghosal, A. (2004). Kinematics of Wheeled Mobile Robots on Uneven Terrain, Mechanism and Machine Theory, Vol. 39, No. 12, (December 2004)12731287, ISSN: 0094-114X

Dai, J. S. \& Jones, J. Rees. (1999). Mobility in Metamorphic Mechanisms of Foldable/Erectable Kinds, ASME Journal of Mechanical Design, Vol. 121, No. 3, (1999)375-382, ISSN: 1050-0472

Gogu, G. (2005a). Mobility of Mechanisms: a Critical Review. Mechanism and Machine Theory, Vol. 40, No. 9, (September 2005) 1068-1097, ISSN: 0094-114X

Gogu, G. (2005b). Mobility and Spatiality of Parallel Robots Revisited via Theory of Linear Transformations, European Journal of Mechanics A/Solids, Vol. 24, No. 4, (JulyAugust 2005) 690-711, ISSN: 0997-7538

Gosselin, C. M. \& Laliberté, C. T. (1998). Underactuated Mechanical Finger with Return Actuation , Patent Number: 5762390, Date of Patent: June 9, 1998, http:/ / robot.gmc.ulaval.ca/en/publi/patents.html.

Huang, Z.; Kong, L. F. \& Fang, Y. F. (1997). Theory of Mechanism of Parallel Robotics and Control, Machinery Industry Press, ISBN:7-111-05812-7, Beijing

Huang, Z.; Zhao, Y. S. \& Zhao, T. S. (2006). Advanced Spatial Mechanism, Advanced Educational Press of China, ISBN:7-04-019265-9, Beijing

Hunt, K. H. (1978). Kinematic Geometry of Mechanisms, Oxford University Press, ISBN 019-856124-5, Oxford

Jain, A. \& Rodriguez, G. (1993) An Analysis of the Kinematics and Dynamics of Underactuated Manipulators, IEEE Transactions on Robotics and Automation, Vol. 9, No. 4, (August 1993)411-422, ISSN: 1042-296X

Kelley, C. T. (1995). Iterative Methods for Linear and Nonlinear Equations, North Carolina State University, Society for Industrial and Applied Mathematics, ISBN : 0898713528(pbk.), Philadelphia

Kempe, A. B. (1877). How to Draw a Straight Line, London: Macmillan. Cited by Henderson, D. W. \& Taimina, D., http://kmoddl.library.cornell.edu/tutorials/11/.

Kumar, V. (1992). Instantaneous Kinematics of Parallel-Chain Robotic Mechanisms, ASME Journal of Mechanical Design, Vol. 114, (September 1992)349-358, ISSN: 0738-0666

Laliberté, T. \& Gosselin, C. M. (1998). Simulation and Design of Underactuated Mechanical Hands, Mechanism and Machine Theory, Vol. 33, No. 1-2, (January-February 1998)39-57, ISSN: 0094-114X

Murakami, T. et al. (1989). Development of a new multi-link front suspension, SAE 890179.

Phillips, J. (1984). Freedom in Machinery, Vol. 1: Introducing Screw Theory, Cambridge University Press, ISBN 0-521-23696-7, Cambridge

Phillips, J. (1990). Freedom in Machinery, Vol. 2: Screw Theory Exemplified, Cambridge University Press, ISBN 0-521-25442-6, Cambridge 
Phillips, J. R. \& Hunt, K. H. (1964). On the Theorem of Three Axes in the Spatial Motion of Three Bodies, Australian Journal of Applied Science, Vol. 15, (1964) 267-287, ISSN: 0572-1156

Raghavan, M. (1996). Number and Dimensional Synthesis of Independent Suspension Mechanisms, Mechanism and Machine Theory, Vol. 31, No. 8, (November, 1996)1141-1153, ISSN: 0094-114X

Raghavan, M. (2005). Suspension Synthesis for N:1 Roll Center Motion, ASME Journal of Mechanical Design, Vol. 127, No. 4, (July 2005)673-678, ISSN: 1050-0472

Rotman, Joseph J. (2002). Advanced Modern Algebra, Prentice Hall, (May 2002) ISBN : 0130878685

Shigley, J. E. \& Uicker, J. J. (1980). Theory of Machines and Mechanisms, New York: McGraw-Hill Companies, Inc. , ISBN: 0070568847 , New York

Simionescu, P. A. \& Beale, D. (2002). Synthesis and Analysis of the Five-Link Rear Suspension System Used in Automobiles, Mechanism and Machine Theory, Vol. 37, No. 9, (September, 2002)815-832, ISSN: 0094-114X

Suh, C. H. (1989). Synthesis and analysis of suspension mechanisms with use of displacement matrices, SAE 890098.

Tsai, L.-W. (2001). Mechanism Design: Enumeration of Kinematic Structures According to Function, CRC Press LLC, ISBN 0-8493-09018, Florida

Waldron, K. J. (1966). The Constraint Analysis of Mechanisms, Journal of Mechanisms, Vol. 1, (1966)101-114, ISSN: 0094-114X

Yan, H.-S. \& Kuo, C.-H. (2006). Topological Representations and Characteristics of Variable Kinematic Joints, ASME Journal of Mechanical Design, Vol. 128, No. 2, (March, 2006)384-391, ISSN: 1050-0472

Yi, B.-J. \& Kim, W. K. (2002). The Kinematics for Redundantly Actuated Omnidirectional Mobile Robots, Journal of Robotic Systems, Vol. 19, No. 6, (June 2002)255-267, ISSN: 0741-2223

Yiu, Y. K. \& Li, Z. (2003). Trajectory Generation for a 2-dof Over-actuated Parallel Manipulator with Actuator Speed and Torque Limits Consideration, Proceedings 2003 IEEE International Symposium on Computational Intelligence in Robotics and Automation, pp.1058-1063, ISBN: 0-7803-7866, July 16-20, 2003, Kobe, Japan.

Zhao, J.-S. et al. (2004a). A New Method to Study the Degree of Freedom of Spatial Parallel Mechanisms. The International Journal of Advanced Manufacturing Technology, Vol. 23, No. 3-4, (February 2004) 288-294, ISSN: 0268-3768

Zhao, J.-S.; Zhou, K. \& Feng, Z.-J. (2004b). A Theory of Degrees of Freedom For Mechanisms, Mechanism and Machine Theory, Vol. 39, No. 6, (June 2004)621-643, ISSN: 0094$114 \mathrm{X}$

Zhao, J.-S. et al. (2006a). Re-analysis of the Degree-of-Freedom Configuration of the Platforms in Spatial Parallel Mechanisms with Constraints Spaces, The International Journal of Advanced Manufacturing Technology, Vol. 28, No. 1-2, (February 2006)190-196, ISSN: 0268-3768

Zhao, J.-S. et al. (2006b). The Free Mobility of a Parallel Manipulator, Robotica, Vol.24, No.5, (September 2006)635-641, ISSN: 0263-5747 
Zhao, J.-S.; Feng, Z.-J. \& Dong, J.- X. (2006c). Computation of the Configuration Degree of Freedom of a Spatial Parallel Mechanism by Using Reciprocal Screw Theory, Mechanism and Machine Theory, Vol. 41, No. 12, (December 2006)1486-1504, ISSN: 0094-114X 


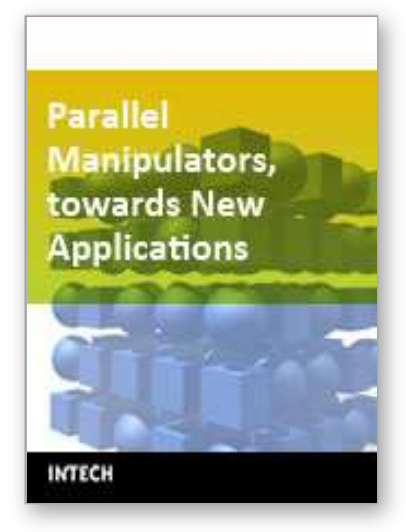

\section{Parallel Manipulators, towards New Applications \\ Edited by Huapeng Wu}

ISBN 978-3-902613-40-0

Hard cover, 506 pages

Publisher I-Tech Education and Publishing

Published online 01, April, 2008

Published in print edition April, 2008

In recent years, parallel kinematics mechanisms have attracted a lot of attention from the academic and industrial communities due to potential applications not only as robot manipulators but also as machine tools. Generally, the criteria used to compare the performance of traditional serial robots and parallel robots are the workspace, the ratio between the payload and the robot mass, accuracy, and dynamic behaviour. In addition to the reduced coupling effect between joints, parallel robots bring the benefits of much higher payload-robot mass ratios, superior accuracy and greater stiffness; qualities which lead to better dynamic performance. The main drawback with parallel robots is the relatively small workspace. A great deal of research on parallel robots has been carried out worldwide, and a large number of parallel mechanism systems have been built for various applications, such as remote handling, machine tools, medical robots, simulators, micro-robots, and humanoid robots. This book opens a window to exceptional research and development work on parallel mechanisms contributed by authors from around the world. Through this window the reader can get a good view of current parallel robot research and applications.

\section{How to reference}

In order to correctly reference this scholarly work, feel free to copy and paste the following:

Jing-Shan Zhao Fulei Chu and Zhi-Jing Feng (2008). Mobility of Spatial Parallel Manipulators, Parallel Manipulators, towards New Applications, Huapeng Wu (Ed.), ISBN: 978-3-902613-40-0, InTech, Available from:

http://www.intechopen.com/books/parallel_manipulators_towards_new_applications/mobility_of_spatial_paralle I_manipulators

\section{INTECH}

open science | open minds

\section{InTech Europe}

University Campus STeP Ri

Slavka Krautzeka 83/A

51000 Rijeka, Croatia

Phone: +385 (51) 770447

Fax: +385 (51) 686166

www.intechopen.com

\section{InTech China}

Unit 405, Office Block, Hotel Equatorial Shanghai

No.65, Yan An Road (West), Shanghai, 200040, China 中国上海市延安西路65号上海国际贵都大饭店办公楼 405 单元

Phone: +86-21-62489820

Fax: +86-21-62489821 
(C) 2008 The Author(s). Licensee IntechOpen. This chapter is distributed under the terms of the Creative Commons Attribution-NonCommercialShareAlike-3.0 License, which permits use, distribution and reproduction for non-commercial purposes, provided the original is properly cited and derivative works building on this content are distributed under the same license. 